\title{
Synthesis and Characterization of Bimetallic Gold-Silver Core-Shell Nanoparticles: A Green Approach
}

\author{
A. Calagua', H. Alarcon'1, F. Paraguay², Juan Rodriguez ${ }^{1}$ \\ ${ }^{1}$ Facultad de Ciencias, Universidad Nacional de Ingeniería, Lima, Perú \\ ${ }^{2}$ Laboratorio Nacional de Nanotecnología, Centro de Investigación de Materiales Avanzados, Chihuahua, \\ México \\ Email: jrodriguez@uni.edu.pe
}

Received 7 August 2015; accepted 6 November 2015; published 9 November 2015

Copyright (C) 2015 by authors and Scientific Research Publishing Inc.

This work is licensed under the Creative Commons Attribution International License (CC BY). http://creativecommons.org/licenses/by/4.0/

(c) (i) Open Access

\begin{abstract}
Bimetallic gold-silver core-shell nanoparticles were prepared by chemical reduction in aqueous solution, following a method that was friendly to the environment, allowing us to use this for medicinal purposes. Gold nanoparticles were synthesized, and silver cations were then reduced on the nanoparticles. Using the optical properties of metallic nanoparticles, surface plasmon resonance was determined by UV-Vis spectroscopy, and the values obtained for gold and silver were approximately $520 \mathrm{~nm}$ and $400 \mathrm{~nm}$ in wavelength, respectively. The absorption peaks of the surface plasmon band show a clear red-shift due to size effect in the case of the silver surface, and a plasmon coupling effect, in the case of gold. To obtain a better understanding of the coating conditions, high resolution transmission electron microscopy was used. The average hydrodynamic size and the size distribution of the synthesized nanoparticles were obtained by dynamic light scattering. The development of this process, which is benign for the environment, opens the possibility for many applications in the areas of renewable energy, medicine and biology.
\end{abstract}

\section{Keywords}

Nanoparticles, Bimetallic, Core-Shell, Gold, Silver, Au/Ag

\section{Introduction}

The size reduction of materials to the nanometric scale brings important effects on their optical properties [1]. The size dependence of these properties falls into two groups: the first one includes those properties related to changes 
in the energy levels of system, passing from a continuous system to a discrete system; and the second one includes those properties related to surface plasmon resonance [1] (SPR), which is the coherent excitation of all the free electrons in the conduction band produced by a beam of incident light [2] [3]. SPR can be influenced by the factors that modify the charge density on the surface of the nanoparticle, such as its morphology, composition, adsorbates, intermolecular distance and dielectric properties of the metal as well as the surrounding medium [3] [4]. Nanoparticles of gold and silver, in particular, have received a lot of attention due to the wavelengths at which SPR occurs, approximately $520 \mathrm{~nm}$ and $400 \mathrm{~nm}$ respectively, which are in the visible range of the electromagnetic spectrum [2].

Silver nanoparticles have been found to have interesting properties in the field of medicine, particularly against fungal and viral infections, inflammation, angiogenesis, platelet aggregation and cancer cells [5] [6]. Gold nanoparticles also have possible therapeutic applications, including activity against HIV, angiogenesis, malaria and arthritis. They can be used for the production of bio-hydrogen [6]-[8]; since the SPR is in the visible range, they have applications for the diagnosis and treatment of cancer, for medical biosensors, drug delivery, DNA analysis, and even as light activated nano-scale heaters [6] [9] [10].

By combining these two metals within the same structure, two possibilities may arise: 1) a single SPR, if both metals combine to form an alloy [11]-[13]; or 2) two continuous and independent SPRs, if they form core-shelltype structures [11] [13] [14]. In both cases, the SPRs are restricted to the range between 520 and $400 \mathrm{~nm}$ [2] [11]-[14], with, for core-shell-type structures, some small shifts affecting the SPR [2]-[4]. These characteristics can lead to interesting applications in optics, as well as for medical diagnoses [3] [4] [6]. They could even be used for photothermal treatments of diseases like cancer [3] [6], using the SPR emitted photos presenting larger energy they absorbed [3].

Various methods have been developed to obtain gold-silver core-shell-type bimetallic nanoparticles, using sodium borohydride $\left(\mathrm{NaBH}_{4}\right)$ [4] [9] [11], hexadecyltrimethylammonium bromide (CTAB) [4] [9], polyacrylamide $\left[\mathrm{C}_{3} \mathrm{H}_{5} \mathrm{NO}\right]_{n}$ [2] [9], hydrazine $\left(\mathrm{N}_{2} \mathrm{H}_{4}\right)$ [2], or glycine $\left(\mathrm{C}_{2} \mathrm{H}_{5} \mathrm{NO}_{2}\right)$ [9]. Unfortunately, all these compounds have a high environmental impact, which would preclude a fortiori their use for medicinal purposes. There is a growing need to develop a green synthesis of nanoparticles [15], therefore, it is proposed in this work to use sodium citrate $\left(\mathrm{Na}_{3} \mathrm{C}_{3} \mathrm{H}_{5} \mathrm{O}(\mathrm{COO})_{3}\right)$ and ascorbic acid $(\mathrm{L})\left(\mathrm{C}_{6} \mathrm{H}_{8} \mathrm{O}_{6}\right)$ as the only reducing agent and stabilizer, as both compounds are innocuous and safe for the environment.

\section{Experimental}

\subsection{Reagents}

Chloroauric acid ( $\mathrm{HAuCl}_{4}$-Sigma-Aldrich, USA, ACS reagent) and silver nitrate ( $\mathrm{AgNO}_{3}$-Merck Peruana SA, Peru, reagent for analysis) were used to prepare the core and the metal layer of the core-shell-type bimetallic nanoparticles. Sodium citrate $\left(\mathrm{Na}_{3} \mathrm{C}_{3} \mathrm{H}_{5} \mathrm{O} \text { (COO) }\right)_{3}$-Sigma, USA), and ascorbic acid (L) $\left(\mathrm{C}_{6} \mathrm{H}_{8} \mathrm{O}_{6}\right.$-Riedel-de Haen AG Seelze-Hannover, reagent for analysis) were used as reducing and stabilizing agents of the synthesized nanoparticles.

\subsection{Instruments}

The surface plasmon resonance of the nanoparticles was measured using a UV-Vis Spectrophotometer (Perkin Elmer LAMBDA 25). The samples were diluted with ultrapure water at a ratio of 1:3 at room temperature (about 20C). The mixture was then introduced in a glass cell with a $1 \mathrm{~cm}$ optical path length. The spectral wavelength range used was from 300 to $800 \mathrm{~nm}$.

The size distribution and the average hydrodynamic size of the synthesized nanoparticles were measured by dynamic light scattering (DLS-Brookhaven 90 Plus). The diluted mixtures of nanoparticles were analyzed using a cell with a $1 \mathrm{~cm}$ optical path length and a LaserMax monochromatic $(658 \mathrm{~nm})$ laser.

The morphology and size of the particles were studied by high resolution transmission electron microscopy (HRTEM) at the National Nanotechnology Laboratory, Research Center for Advanced Materials, Chihuahua, Chihuahua, Mexico.

\subsection{Synthesis of Gold Nanoparticles (Seeds)}

To prepare the gold nanoparticles, $10 \mathrm{~mL}$ of $\mathrm{HAuCl}_{4}(1 \mathrm{mM})$ and $4 \mathrm{~mL}$ of water were brought to a boil, then $6 \mathrm{~mL}$ 
of a solution of sodium citrate (35 mM) were added, maintaining the mixture under reflux for $15 \mathrm{~min}$. With time, a change in the color of the solution from yellow to red-brown was observed. Once the reflux time was completed, the solution was allowed to cool for 30 minutes at room temperature and then for 30 minutes more in an ice bath.

\subsection{Silver Coating of the Gold Seeds}

In a $125 \mathrm{~mL}$ flask, $20 \mathrm{~mL}$ of water and $0.1 \mathrm{~mL}$ of ascorbic acid $(0.01 \mathrm{M})$ were mixed and kept under stirring. Then, $300 \mu \mathrm{L}$ of the gold seed sol were added and stirred for 2 minutes. Finally, while still stirring, $30 \mathrm{~mL}$ of silver nitrate $(0.01 \mathrm{M})$ were added drop by drop and stirring was continued for 30 minutes at room temperature.

\section{Results and Discussion}

\subsection{Synthesis of Gold Nanoparticles (Seeds)}

To form nanoparticles by homogeneous nucleation, reduction of the $\mathrm{Au}^{3+}$ ion must take place, followed by an aggregation process of these reduced species. The reduction of $\mathrm{Au}^{3+}$ ion to Au transforms a soluble cation into an insoluble metal atom, generating solid atomic species in a liquid medium. This process produces a large surface energy due to the high segregation of atomic species, which destabilizes the system and promotes the formation of colloidal aggregates. Since low precursor concentrations were used, the diffusion of gold atoms on the nanoparticle surface takes place slowly and directionally.

The number of particles with enough energy to overcome the critical energy barrier increases with temperature. For this reason, the synthesis of gold nanoparticles was carried out at the boiling point of the mixture (see Table 1).

Nanoparticles are metastable due to their high surface to volume ratio compared to bulk material and must be stabilized kinetically, since they are not thermodynamically stable. Stabilization in this case was accomplished using sodium citrate, which is electrostatically bonded to the surface of the nanoparticles by cationic intermediates, and generates electrostatic repulsion through its surface charge. Decreasing the amount of stabilizing agent or the surface charge will cause the particles to form aggregates and possibly precipitate.

The SPR value obtained for the gold nanoparticles was $521.4 \mathrm{~nm}$, a value consistent with the Mie theory (see Table 1 and Figure 1).

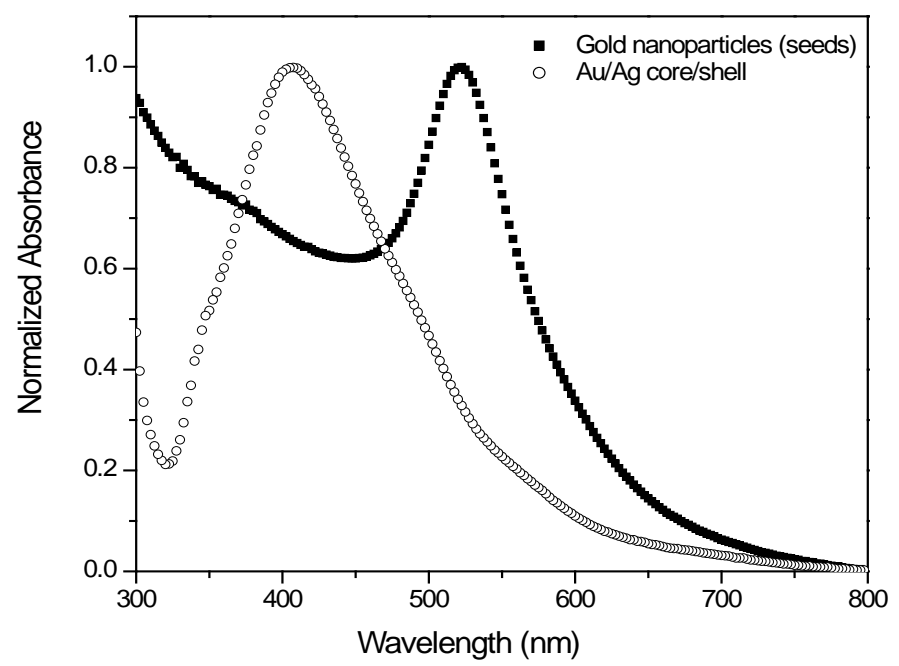

Figure 1. Normalized UV-Vis spectrum for (a) Au nanoparticles and (b) Au/Ag core-shell-type bimetallic nanoparticles.

Table 1. Comparison of results obtained with HRTEM, DLS y UV-Vis.

\begin{tabular}{|c|c|c|c|c|c|c|}
\hline \multirow{2}{*}{ Nanopartícles } & \multirow{2}{*}{$\begin{array}{l}\text { Hydrodynamic } \\
\text { diameter (nm) }\end{array}$} & \multirow{2}{*}{ Polydispersity index } & \multicolumn{2}{|c|}{ Wavelength. (nm) } & \multicolumn{2}{|c|}{ Diameter-HRTEM (nm) } \\
\hline & & & $\mathrm{Au}$ & Ag & Inside diameter & Outside diameter \\
\hline $\mathrm{Au}$ & 40.1 & 0.282 & 521.4 & - & - & - \\
\hline $\mathrm{Au} / \mathrm{Ag}$ & 64.3 & 0.323 & 570 & 407.6 & 22.3 & 25.6 \\
\hline
\end{tabular}




\subsection{Silver Coating of the Gold Seeds}

The presence of gold seeds causes a controlled reduction (B) of the silver salt [16], through a process of heterogeneous nucleation on the surface of the gold nanoparticles.

The conditions for the deposition of the silver layer were different from those used for the synthesis of the gold seeds. In this case, the system is not exposed to high temperature as before, so that the activation energy for nucleation of the solid phase is smaller. Since the energy barrier is higher, the number of silver nuclei capable to overcome this barrier will be lower, migrating to reduce the total free energy, generating growth on the surface of gold seeds.

The $\mathrm{pH}$ of the mixture of ascorbic acid and gold nanoparticles was below 6, so that the equilibrium in Equation (1) is shifted towards the formation of citric acid.

$$
\mathrm{C}_{3} \mathrm{H}_{5} \mathrm{O}\left(\mathrm{COO}^{-}\right)_{3}+3 \mathrm{H}^{+} \leftrightarrow \mathrm{C}_{3} \mathrm{H}_{5} \mathrm{O}(\mathrm{COOH})_{3}
$$

This in turn partially unprotects the gold NPs, and leave them exposed for the second growth stage, during which ascorbic acid is oxidized and silver is reduced, forming metallic silver. At a later stage, ascorbic acid may be degraded by decarboxylation and the silver takes over as growth agent for the nanoparticle, so as to obtain the desired core-shell structure.

The silver ions, in presence of ascorbic acid, are reduced to silver atoms, producing dehydroascorbate, itself hydrolyzed to 2, 3 diketogulonic acid, and eventually decomposed by decarboxylation. The process is controlled by diffusion, thanks to the constant stirring provided to the system.

The processes occurring during particle growth through reduction are conventionally described by the following steps: 1) diffusion of the growth species to the surface; 2) adsorption of these species; and 3) irreversible incorporation of these species to the surface (the growth step itself), where stable metal-metal bonds are formed. This latter step is the most important as it provides the shape of the coating.

As can be seen in Figure 2, the coating is fairly homogeneous. Since deposition was carried out at low concentrations, the growth process is expected to be diffusion controlled. However, one can expect that on the surface of the nanoparticle, some surface phenomena will take place, as the nanoparticles, aside from being spherical, have a polydispersity between $28 \%$ and $32 \%$ (see Table 1 ).

The value of the SPR wavelength for silver in the core-shell structure was found to be $407.6 \mathrm{~nm}$, which is within the expected range of $400 \mathrm{~nm}$ to $410 \mathrm{~nm}$ (see Figure 1 and Table 1).

Comparing the SPR of gold as seed and as part of the core-shell structure (Figure 1), one can see a red shift. This can be due to the existence of a region between core and shell where mixing takes place [3] [13]. This phenomenon would depend upon the coating thickness, and generate its own oscillation, coupled to those of the inner and outer surfaces. Mixing can be explained by the similar lattice parameters of gold $(0.408 \mathrm{~nm})$ and silver $(0.409 \mathrm{~nm})$ and their similar atomic radii (0.144 nm for both).

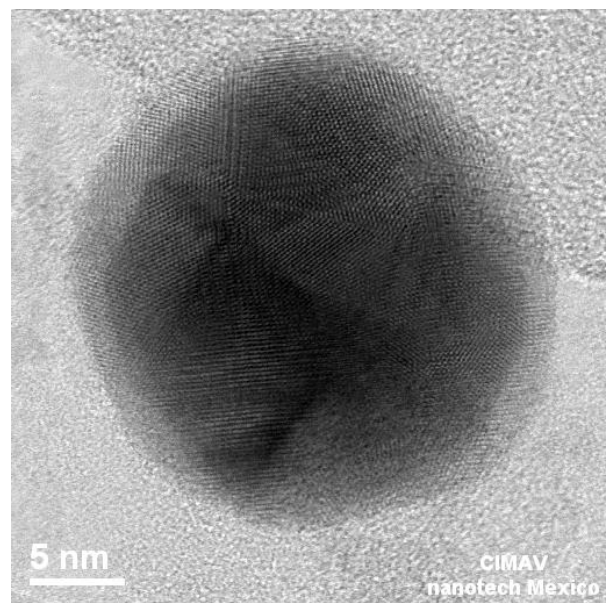

(a)

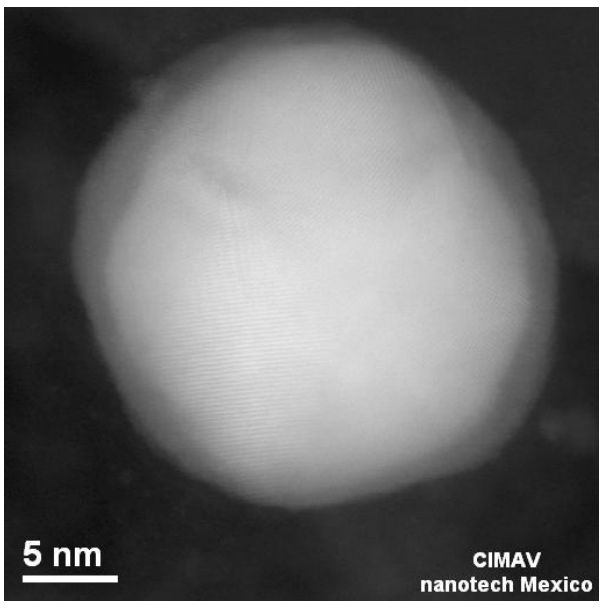

(b)

Figure 2. Gold-silver core-shell nanoparticle as examined with (a) bright field and (b) dark field HRTEM. 
The polydispersity found for the core-shell structures differs from that observed for the seeds (Table 1). This could be expected as a small number of silver nanoparticles may nucleate during the coating operation, increasing slightly polydispersity.

\section{Conclusions}

Gold nanoparticles were synthesized with a $40.1 \mathrm{~nm}$ mean hydrodynamic diameter, as well as gold-silver core-shell nanoparticles with a $64.3 \mathrm{~nm}$ mean hydrodynamic diameter and a $25.7 \mathrm{~nm}$ average diameter, as estimated by HRTEM.

The surface plasmon resonances obtained for the gold nanoparticles and the gold core of gold-silver core-shell nanoparticles were $521.4 \mathrm{~nm}$ and $570.0 \mathrm{~nm}$ respectively (see Table 1), showing the importance of the silver coating on the gold core.

The SPR of the gold-silver core-shell nanoparticles show two peaks at $407.6 \mathrm{~nm}$ and $570 \mathrm{~nm}$, demonstrating the possibility of controlling the optical properties of gold and silver nanoparticles for applications in counter electrodes for solar cells. Such a work is currently under development.

\section{Acknowledgements}

The present research has been funded by the Fincyt Project No. 140-FINCYT-IB-2013, the Research Institute of the National University of Engineering-Faculty of Science and by the CONCYTEC Chair in Nanomaterials.

\section{References}

[1] Lee, Y.S. (2008) Self-Assembly and Nanotechnology. John Wiley \& Sons, Inc., Hoboken.

[2] Pal, A., Shah, S. and Devi, S. (2007) Synthesis of Au, Ag and Au-Ag Alloy Nanoparticles in Aqueous Polymer Solution. Colloids and Surfaces A: Physicochemical and Engineering Aspects, 302, 51-57. http://dx.doi.org/10.1016/j.colsurfa.2007.01.054

[3] Jain, P.K., Huang, X.H., El-Sayed, I.H. and El-Sayed M.A. (2008) Noble Metals on the Nanoscale: Optical and Photothermal Properties and Some Applications in Imaging, Sensing, Biology, and Medicine. Accounts of Chemical Research, 41, 1578-1586. http://dx.doi.org/10.1021/ar7002804

[4] Petryayeva, E. and Krull, U.J. (2011) Localized Surface Plasmon Resonance: Nanostructures, Bioassays and Biosensing-A Review. Analytica Chimica Acta, 706, 8-24. http://dx.doi.org/10.1016/j.aca.2011.08.020

[5] Sheny, D.S., Mathew, J. and Philip, D. (2011) Phytosynthesis of Au, Ag and Au-Ag Bimetallic Nanoparticles Using Aqueous Extract and Dried Leaf of Anacardium occidentale. Spectrochimica Acta Part A, 79, 254-262. http://dx.doi.org/10.1016/j.saa.2011.02.051

[6] Larguinho, M. and Baptista, P.V. (2012) Gold and Silver Nanoparticles for Clinical Diagnostics—From Genomics to Proteomics. Journal of Proteomics, 75, 2811-2823. http://dx.doi.org/10.1016/j.jprot.2011.11.007

[7] Kalishwaralal, K., Deepak, V., Pandian, S.B.R.K., Kottaisamy, M., Kanth, S.B.M., Kartikeyan, B. and Gurunathan, S. (2010) Biosynthesis of Silver and Gold Nanoparticles Using Brevibacterium casei. Colloids and Surfaces B: Biointerfaces, 77, 257-262. http://dx.doi.org/10.1016/j.colsurfb.2010.02.007

[8] Zhang, Y.F. and Shen, J.Q. (2007) Enhancement Effect of Gold Nanoparticles on Biohydrogen Production from Artificial Wastewater. International Journal of Hydrogen Energy, 32, 17-23. http://dx.doi.org/10.1016/j.ijhydene.2006.06.004

[9] Tran, T.-H. and Nguyen, T.-D. (2011) Controlled Growth of Uniform Noble Metal Nanocrystals: Aqueous-Based Synthesis and Some Applications in Biomedicine. Colloids and Surfaces B: Biointerfaces, 88, 1-22. http://dx.doi.org/10.1016/j.colsurfb.2011.07.017

[10] Endo, T., Ikeda, D., Kawakami, Y., Yanagida, Y. and Hatsuzawa, T. (2010) Fabrication of Core-Shell Structured Nanoparticle Layer Substrate for Excitation of Localized Surface Plasmon Resonance and Its Optical Response for DNA in Aqueous Conditions. Analytica Chimica Acta, 661, 200-205. http://dx.doi.org/10.1016/j.aca.2009.12.022

[11] Chen, H.M., Liu, R.S., Jang, L.-Y., Lee, J.-F. and Hu, S.F. (2006) Characterization of Core-Shell Type and Alloy Ag/ Au Bimetallic Clusters by Using Extended X-Ray Absorption Fine Structure Spectroscopy. Chemical Physics Letters, 421, 118-123. http://dx.doi.org/10.1016/j.cplett.2006.01.043

[12] Sun, L., Luan, W.L., Shan, Y.J. and Tu, S.-T. (2012) One-Step Synthesis of Monodisperse Au-Ag Alloy Nanoparticles in a Microreaction System. Chemical Engineering Journal, 189-190, 451-455. http://dx.doi.org/10.1016/j.cej.2012.02.043 
[13] Shibata, T., Bunker, B.A., Zhang, Z.Y., Meisel, D., Vardeman II, C.F. and Gezelter, J.D. (2002) Size-Dependent Spontaneous Alloying of Au-Ag Nanoparticles. Journal of the American Chemical Society, 124, 11989-11996. http://dx.doi.org/10.1021/ja026764r

[14] Fratoddi, I., Venditti, I., Battocchio, C., Polzonetti, G., Cametti, C. and Russo, M.V. (2011) Core Shell Hybrids Based on Noble Metal Nanoparticles and Conjugated Polymers: Synthesis and Characterization. Nanoscale Research Letters, 6, 98. http://dx.doi.org/10.1186/1556-276X-6-98

[15] Raveendran, P., Fu, J. and Wallen, S.L. (2003) Completely “Green” Synthesis and Stabilization of Metal Nanoparticles. Journal of the American Chemical Society, 125, 13940-13941. http://dx.doi.org/10.1021/ja029267j

[16] Jana, N.R., Gearheart, L. and Murphy, C.J. (2001) Evidence for Seed Mediated Nucleation in the Chemical Reduction of Gold Salts to Gold Nanoparticles. Chemistry of Materials, 13, 2313-2322. http://dx.doi.org/10.1021/cm000662n 